\title{
The Crux of Integration Prototyping in Complex Environments
}

\author{
A case study of integration prototyping with a low-cost sonar leakage system
}

\author{
Johan Chr. W. Hjorth ${ }^{1}$, Heikki Sjöman ${ }^{1}$, Martin Steinert ${ }^{1}$ \\ ${ }^{1}$ Norwegian University of Science and Technology \\ jchjorth@stud.ntnu.no \\ heikki.sjoman@ntnu.no \\ martin.steinert@ntnu.no
}

\begin{abstract}
This paper considers the application field of testing a prototype in prototyped test environments versus in the actual intended environment. The research question is how to use prototyped test environments the best possible way in the early stage product development projects where the intended environment is complex. This is done by studying a project with the aim of developing a new low-cost concept to locate water pipe leakages but with inconclusive results. The concept lets a passive sonar flow in the water stream in a pipe, and measures the distance traveled with a pinger positioned at a known point. The pipe network is defined as a complex environment in that it is inaccessible, except for professionals with special training. The two key variables in this concept that together determine a leakage's location are the distance from the start point to the sonar, and the sound pressure level (SPL) ambient to the sonar. Wayfaring with a bias toward building and testing have been used within a framework of engineering design. Three subsystems were designed, built, and tested separately to meet individual requirements set from the start, as well as emerging requirements. When the individual requirements were met, the subsystems were integrated into more relevant test environments. The final prototype has been tested to be able to measure the two key variables. Although the distance measurement is accurate and has a sufficient range in an underwater environment, the results are not equal when tested in the real pipe-system. We wanted to share a snapshot of our system in a realistic development case before taking the project further illustrating the real problems developers face during projects. A focus on prototyping test environments with the aim to test the solution's performance to different specific variables of the intended environment may have been an efficient way to capture the knowledge necessary to develop a successful solution. However, we argue that in order to unveil what relevant attributes that are different between a test environment and the intended environment to our solution, one must test the solution in the actual environment as well. We therefore, suggest alternating approach of testing in the intended environment and prototyped test environments. This paper illustrates the value of developing prototypes with a low resolution so that critical variables of the problem and solution space, and other negative consequences, can be illuminated, with a minimum amount of time and resources.
\end{abstract}




\section{Introduction}

In this paper we will describe on our experience with the relationship between prototyping test setups to fit specific test variable as (Winjum et al. 2017 Welo, \& Steinert, 2017) suggests, and comparing the results with integrated testing in the actual environment. The emerged opportunity from this study was to understand how to find the variables that play the key role in development process when prototyping for complex environments and what of this can be generalized. We do this by analyzing a real-life development project done by the authors in our development laboratory resolving a real need from the industry. The emerged research question is how to use prototyped test environments the best possible way in the early stage product development projects where the intended environment is complex.

In 2017 a report (Norsk Vann 2017) on the quality of the 81 Norwegian municipalities' water and sewage systems was published. Out of the 81 participating municipalities, $9 \%$ have a water loss of less than $20 \%$, and $40 \%$ of the municipalities have a water loss higher than 40 $\%$. Leakages may also cause drops in pressure, which again may cause contaminants to enter the distribution network. Contaminated water can be dangerous and may cause sickness and even death. This is not sustainable in terms of health, environment, or economy. Many of the techniques used to locate leakages today are based on the iron pipes' acoustic abilities, which is less effective when handling newer polymer pipes that are implemented today. In this development project, we have aimed to develop a new low-cost method to locate leakages in water pipes. The research questions for the case project were as follows:

- May we observe water pipe leakages with a low-cost self-made audio sensor?

- Is measuring the distance with acoustic time-of-flight a possible solution with lowcost equipment?

- If so, is the accuracy and range of the solution satisfactory?

\subsection{Concept}

From a pre-project where several ways of measuring distances underwater in low-cost manners were explored, a concept that could be applied in locating leakages emerged. Our solution is to measure the mechanical waves that propagate through the liquid as a result of a leakage in the pressurized pipe. Instead of statically detect leakages with hydrophones or microphones, a hydrophone is put inside the cold $\left(3.5^{\circ} \mathrm{C}\right)$ and pressurized $(7.3$ bar $)$ pipe. By letting the water carry the sonar through the pipeline, long stretches of the pipeline may be inspected effectively, and leakages, as well as other data, may be detected accurately $(<1$ meter). We benchmarked a similar solution, that uses a different measuring principle for determining the location of leakages (Pure 2020). The overview of our concept is illustrated in the Fejl! Henvisningskilde ikke fundet.. 1. 


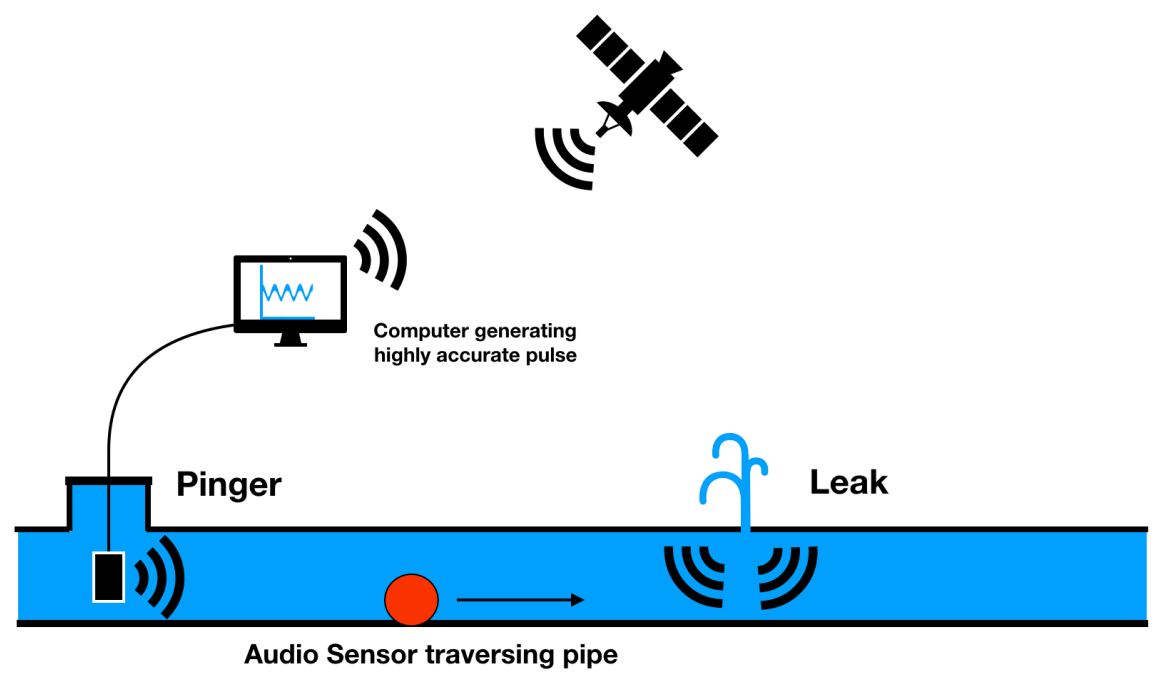

Figure 1.1

One of the biggest challenges with this concept is not to detect leakages but the ability to determine the location of the sonar at the point in time the leak was detected. We have separately solved this by having a pulse sent out from the start position with very accurate rhythm. By knowing the speed of sound in the medium as well as the point in time, the signal is emitted and received, the distance between the pinger and the sensor package can be calculated.

\section{Method}

The research method used to create the case was participatory action research as described by (Ottosson 2003). Furthermore, we have in this project used the methodology of (Yin 2017) and (Eisenhardt) for building theory from case studies.

In this project, we have used a framework of Engineering design by (Pahl and Beitz 2013). The general guidelines of engineering design research of (Blessing and Chakrabarti 2009) are also followed. Having a clear vision of how the concept should materialize, the system and subsystems have been designed and tested to meet requirements set from the start. This has been followed by more complex tests, testing the integrated system as a whole, which has generated emerging requirements.

Within this framework, an exploratory approach with an emphasis on testing to learn has been used to gain knowledge of problem space and concept at hand, namely, the Wayfaring Method that was described by (Gerstenberg et al. 2015 Abrahamsson, \& Steinert, 2015), which again was based on the Hunter-Gatherer Model introduced by (Steinert and Leifer 2012). This method can be visualized as a map where the end-point changes as the path is explored along the way, as an analogy to the old wayfaring way of traveling. The direction of where to go next is guided by gained knowledge from newly probed ideas. By building rapid prototypes and testing them in design-build-test cycles, ideally, a team with a diverse skill set probes the solution space. The aim of these prototypes is to rapidly learn more about their current surroundings in the solution space, but it is not to make perfect solutions. Letting insights guide the product developer through the solution space in this way can lead you to unexpected twists and turns, which may help you uncover 'the big next idea' and find unknown unknowns as referred to by (Gerstenberg et al. 2015, Sutcliffe and Sawyer 2013) . As one learns more about the problem and possible solutions through this journey, one can, in addition, set dynamic requirements (Kriesi et al. 2016). (Kriesi et al. 2016 \& Steinert, 2016) 
propose that this way of letting the insights from exploring to set a part of the requirements may generate more radical and innovative solutions

The design approach used in this project have been of an exploratory nature, where prototyping leads the way within the framework of engineering design (Schrage 1996). With a strong bias toward building and testing, knowledge and insights about the problem and solutions have been acquired (Schón 1983). The set-based mindset will also be a part of the overall approach with prototypes that allow separate building and testing of subsections and configurations (Kennedy, Sobek, and Kennedy 2014).

\section{Development path}

Through interviewing Trondheim Vann og Avløp a clear requirement for a leakage detection tool was formulated as follows: The low-cost system must be able to identify leaks in 6" wide and polyethylene pipe stretches with at least $1 \mathrm{~m}$ accuracy. To meet these requirements with our concept, sets of start-requirements were set for the different subsystems. The different modules were designed built and tested concurrently. The start-requirements for the three different modules are listed below, with a quick description of the development, followed by requirements for the integrated system. It was chosen to divide into these subsystems because they in themselves had clear requirements that would have to be met individually. It was, therefore, effective to develop and test them concurrently. We define an integration prototype as integrating several prototyped subsystems together as a one functioning prototype that is ought to test whether all the requirements are still fulfilled after integration and implementation of learnings from the previous prototypes (Gerstenberg et al. 2015).

\subsection{Sonar Transducer requirements:}

Main requirement: The Sonar Transducer needs to be sensitive enough to detect leakages and pinger pulses while carrying all electronics in a robust and watertight way.

- It must be able to register relevant frequencies from leakages and pinger

- It must be able to contain all required electronics for the sonar physically.

- It needs to be able to roll in all three axes inside a 6" pipe.

- It must be e watertight at least at 10 bar.

- It must have a total higher density than ambient water so that it will sink in water.

- The noise created from motion cannot saturate the transducer.

The sonar transducer was created from the ground up in this project. An aluminum cylinder was lathed to a thin shell with a welded lid in the bottom. On the outside of the cylinder, five ceramic rings that vibrate radially when subjected to sound were mounted with connected wires. The cylinder was then cast in epoxy, and a PVC lid was lathed. The transducer with the lid was tested to be watertight in a pressure chamber at 20 bar. To map the sensitivity of the transducer do different frequencies and determine its receiving sensitivity at relevant frequencies, it was tested and analyzed. Through testing, it was found that it met all the start requirements.

\subsection{Sonar Electronics requirements:}

Main requirement: The Sonar Electronics needs to be able to record all relevant data for the duration of an inspection.

- It needs to fit inside the sonar Transducer.

- It needs to record autonomously for at least three hours.

- It cannot overheat in three hours

- Battery life needs to be at least three hours 
- File-size and storage capacity needs to fit three hours of recording

- The data needs to be stored in such a way that it is possible to calculate distance accurately from it.

- Abrupted loss of power or other malfunctions should compromise as little recorded data as possible.

- It needs to record an accurate time-pulse from a GPS and record signals from the transducer simultaneously.

- The GPS needs to be able to be synchronized before and after entering the pipe.

- Needs to amplify signals from transducer if necessary.

To record and store the data from the transducer, a Raspberry Pi 3 A+ was used to. A circuit to amplify the signal from the transducer was also made. Originally two lithium batteries were used, but iterative testing revealed that a simple power-bank battery was a more effective way to power the electronics. A Ublox LEA M8T was used as the GPS unit. To convert the analog signal to digital, an Xrtyfy SC1 soundcard with one channel was used. The recording script, parameters such as GPS-ping and the physical layout of the circuit, was developed iteratively as our knowledge grew through testing.

\subsection{Pinger Requirements:}

Main requirement: The Pinger needs to emit an accurate pulse that reaches the sonar in a pipe.

- The pinger needs to be able to emit a mechanical pulse through at least $150 \mathrm{~m}$ in water.

- The frequency of pulse needs to make sense in terms of pipe dimension and transducer.

- The amplitude of the pulse needs to be amplified to a necessary degree.

- The pinger needs to communicate with satellites to ensure accurate triggering of the pulses.

- The pinger needs to be able to be firmly mounted inside the pipe at the center of the pipe cross-section.

- The pipe mount needs to be watertight at 10 bar and let a cable go from the inside to the outside of the closure.

- The resolution distance measurement needs to be at least $1 \mathrm{~m}$ with the sonar moving at $1 \mathrm{~m} / \mathrm{s}$.

For the pinger a transducer with unknown parameters was provided by Norbit Group AS. We tested and analyzed the pinger in order to map the sensitivity of the transducer, do different frequencies and determine its transmitting sensitivity at relevant frequencies. Initially $11 \mathrm{kHz}$ was used to fit the pipe-diameter, but iterative testing revealed that $15 \mathrm{kHz}$ was a better choice for our pinger and environment. The mount was constructed from the ground and was fixed to a lid used in pipe armature used by the water distribution network of the city. The pinger was tested to be watertight at 20 bar in a pressure chamber. An Analog Discovery 2 was used to generate desired pulses, and the scope was used for diagnostics of the whole prototype. A typical amplifier for sound-systems in cars was used to amplify the signal as well as a transformer. A Ublox LEA M8T GPS unit was used in this module as well to trigger the pulse generator.

\subsection{Integrated requirements:}

- The sonar needs to detect leakages from inside of the water distribution network. (Leakage Test) 
- It must be possible to derive the distance between the pinger and the sonar accurately underwater from the gathered data. (Under Water Distance Measurement Test)

- The pinger needs to emit a pulse that reaches the sonar through a pipe stretch in the water distribution network. (Under Water Distance Measurement Test in Actual Environment)

\section{Integration Tests}

\subsection{Leakage Test}

To test if the sonar was able to detect leakages, the sonar was put in water filled pipes with 3.5 degree $C$ and 7.3 bar. Leakages was simulated by opening a valve to release a tiny flow of water from the system resulting in the sound signature of a typical leakage. This is the way the professionals simulate leakages when they are training.

In figure 4.1 a screenshot from the Matlab Signal Analyzer can be seen. The bottom part displays the whole signal in the time domain. The top part show the power spectrum in the frequency domain. It displays the amount each frequency from zero to $22050 \mathrm{~Hz}$ is represented on average for the chosen time section of the signal, which in this case is approximately nine seconds. The section in the middle is a combination of the one at the top and the one at the bottom. Time is on the x-axis, frequency is on the y-axis and the intensity at a small section in time is represented by color.
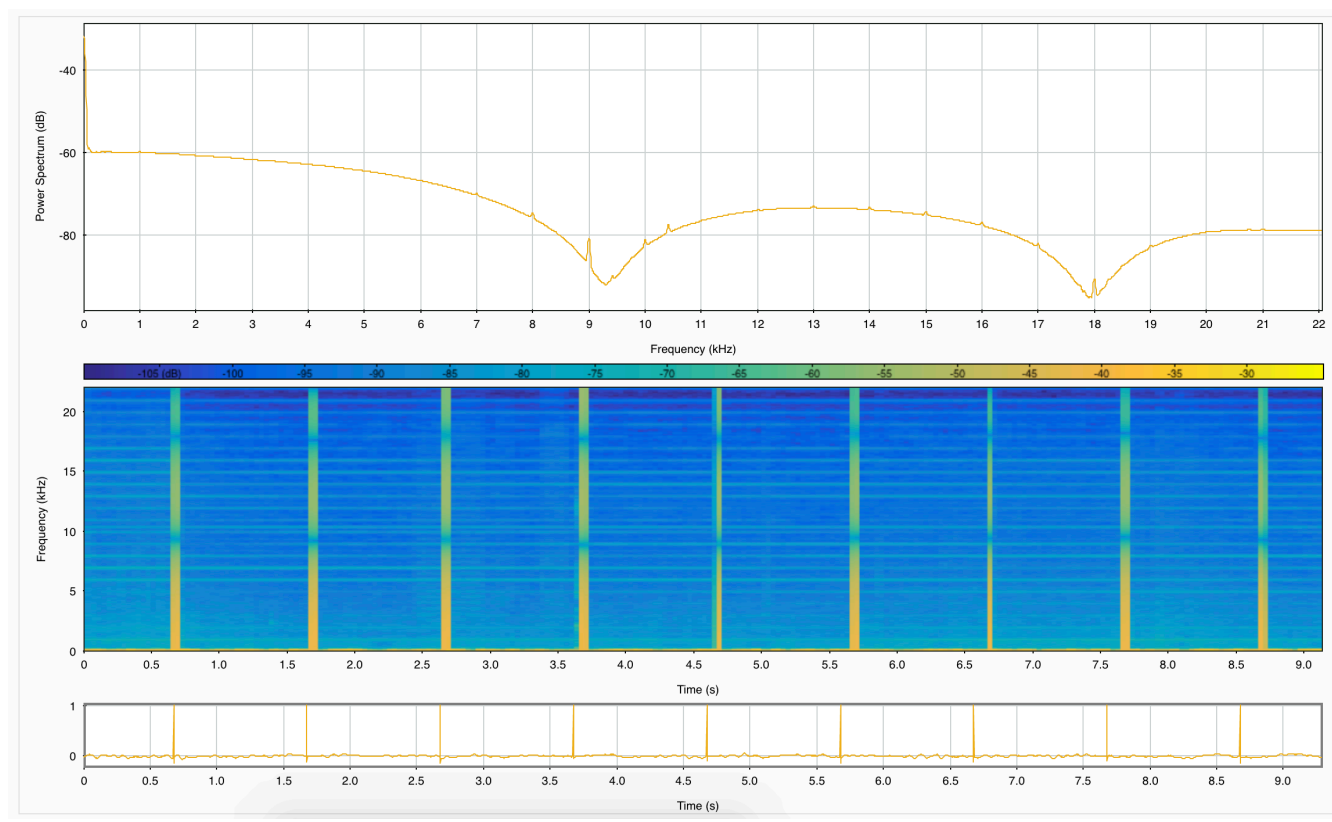

Figure 4.1 


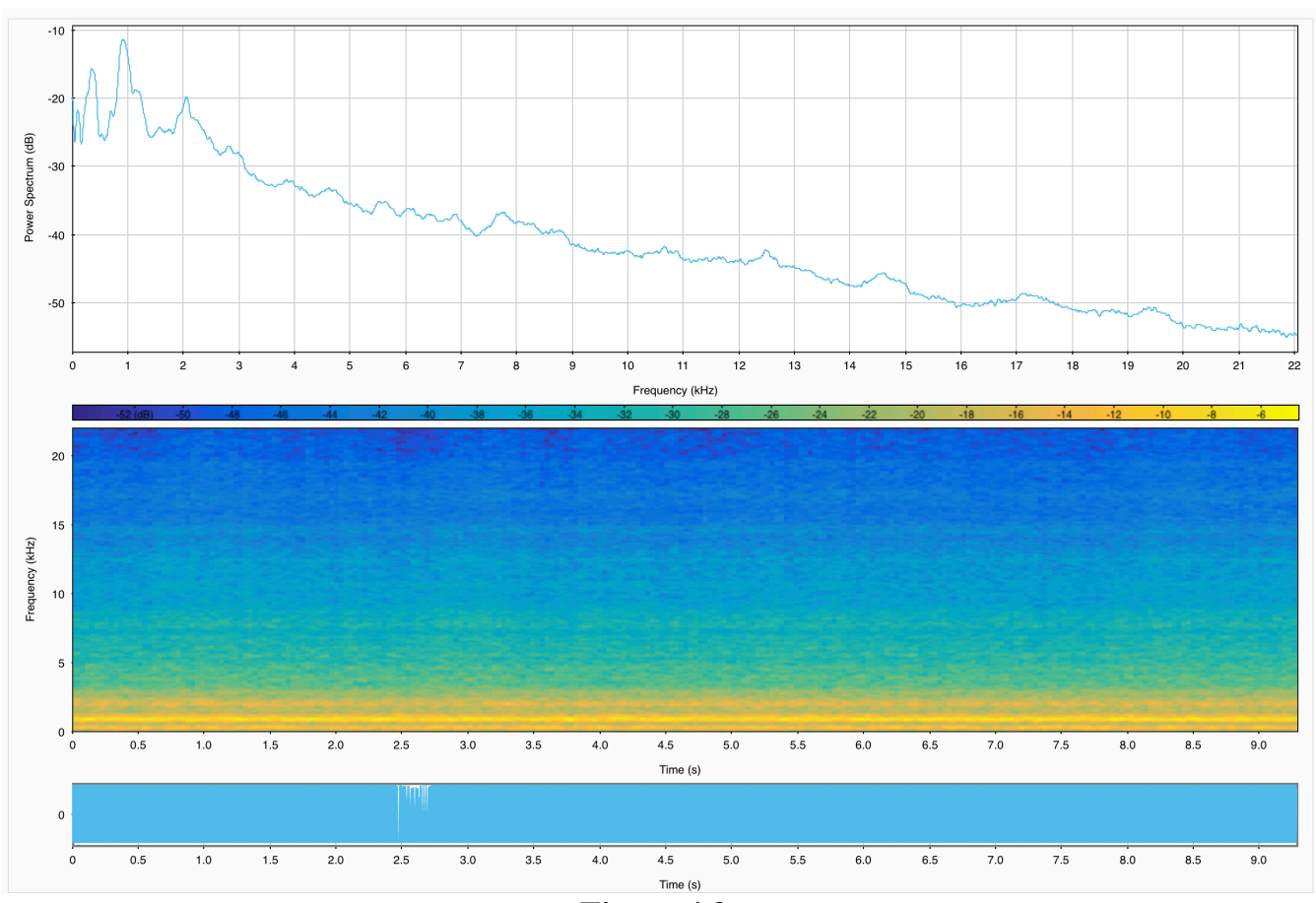

Figure 4.2

In figure 4.2 the recording of the simulated leakage at $0.5 \mathrm{~m}$ in the Matlab Signal Analyzer tool can be seen. Compared to figure 4.1, an obvious increase in amplitude across the whole time sequence can observed. The lower frequencies increase the most. When listening to them with the human ear, one can easily distinguish them, and professionals recognize the sound as a definite leakage. The signals are off the scales which means that the sonar-transducer is saturated and that the amplitude of the alternating pressure is more than $1.2 \mathrm{kPa}$. Theoretically this means that we should be able to detect the leakages more than $3.5 \mathrm{~m}$ away. A leakage was also simulated at $156.3 \mathrm{~m}$, but no recognizable signal was recorded by the sonar. The conclusion of the test is that it is possible to clearly distinguish when the sonar is close to a leakage and when it is not. We can also conclude that $156.3 \mathrm{~m}$ is too far to detect a typical leakage for the sonar.

\subsection{Under Water Distance Measurement Test}

The aim of this test was to determine if the full system was able to measure distances under water, as well as become familiar with the characteristics of the pinger pulse and how it was received by the sonar. This knowledge used to develop a script to calculate distances from the recorded files. This test was performed in an inside pool with fresh water. The pool was approximately $10 \mathrm{~m}$ long, $5 \mathrm{~m}$ wide and $3 \mathrm{~m}$ deep, and the tests were performed at distances of $8.55 \mathrm{~m}$ and $2.54 \mathrm{~m}$, and the depth of approximately $0.5 \mathrm{~m}$. 


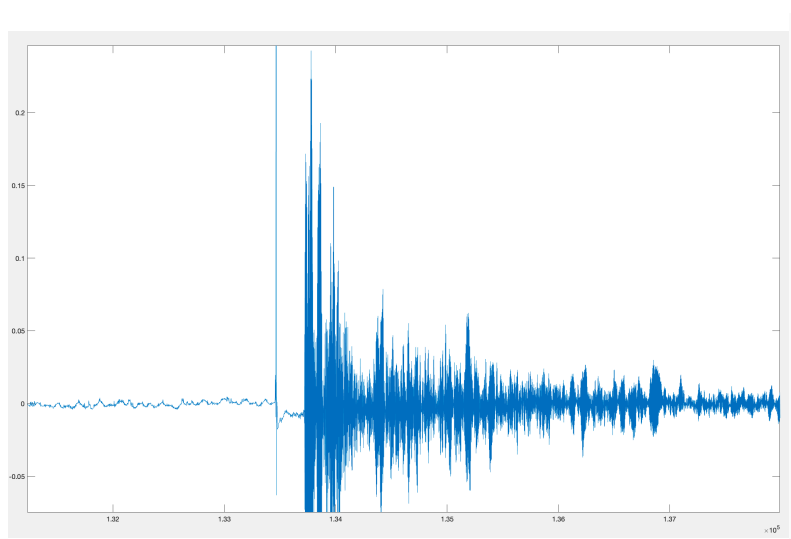

Figure 2.3

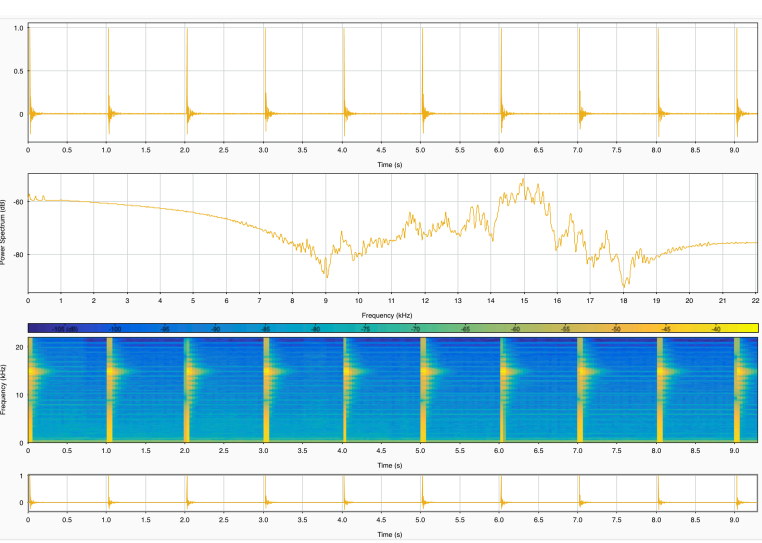

Figure 4.4

In figure 4.3 a closer look at one of the recorded pinger-pulses can be seen. The y-axis is the digital value of where 1 is $230 \mathrm{mV}$, and the $\mathrm{x}$-axis is the sample number. The first amplitude spike from the left is the GPS in the sonar giving setting a timestamp. After a small gap a different signal with a high amplitude is recorded. This is the pinger-pulse reaching the sonar followed by different echoes from the pool. Calculating the difference in time of the timestamp and the onset of the pinger-pulse gives the distance between the two units, given that the GPSes are in sync and the speed of sound in the medium is known.

In figure 4.4 the recording can be seen in Matlabs Signal Analyser tool. One can clearly see the presents of the $15 \mathrm{kHz}$ pinger pulse in the recording. From this test we have learned that the system is capable of performing more than sufficiently accurate measurements under water. The signal strengths measured at different distances also indicates that the prototype will be able to measure longer distances such up to at least $4 \mathrm{~km}$ assuming that the signal decreases with 1/R. The results of the different measurements are listed below.

\begin{tabular}{|l|r|r|r|r|}
\hline Filename & Signal strength Vout [V] & Distance [m] & Measured [m] & Amplitude of pulse \\
\hline 1VLangAvs.wav & 1,0 & 8,55 & 8,5375 & 0,1598 \\
\hline 2VLangAvs.wav & 2,0 & 8,55 & 8,5541 & 0,2234 \\
\hline 4VLangAvs.wav & 4,0 & 8,55 & 8,5560 & 0,3799 \\
\hline 1VLangAvstand.wav & 1,0 & 8,55 & 8,5409 & 0,1174 \\
\hline HalvVoltLangAvstand & 0,5 & 8,55 & 8,5818 & 0,05289 \\
\hline Avstandsmåling16031V & 1,0 & 2,52 & 2,5247 & 0,4563 \\
\hline Avstandsmåling16062V & 2,0 & 2,52 & 2,4730 & 0,6283 \\
\hline
\end{tabular}

\subsection{Under Water Distance Measurement Test in actual Environment}

With the aim to determine if the prototype could do measurements in the actual environment the concept is intended for, and learn more of how the pinger pulse traversed an actual pipe, we were able to perform a test at a pipe-stretch in the water distribution network of the city. The pressure and temperature in the pipe on the test day was 7.3 bar and 3.5 degrees Celsius respectively. The material of the pipe is polyethylene and it has diameter of $200 \mathrm{~mm}$. 


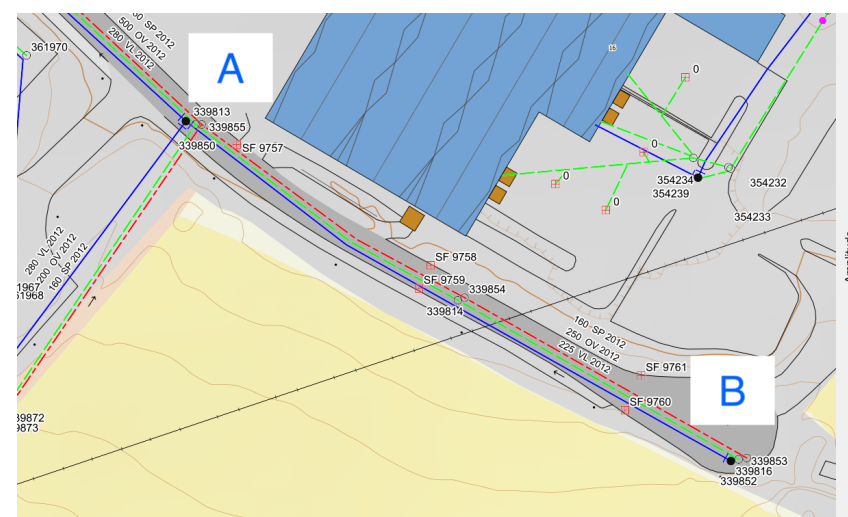

Figure 4.5

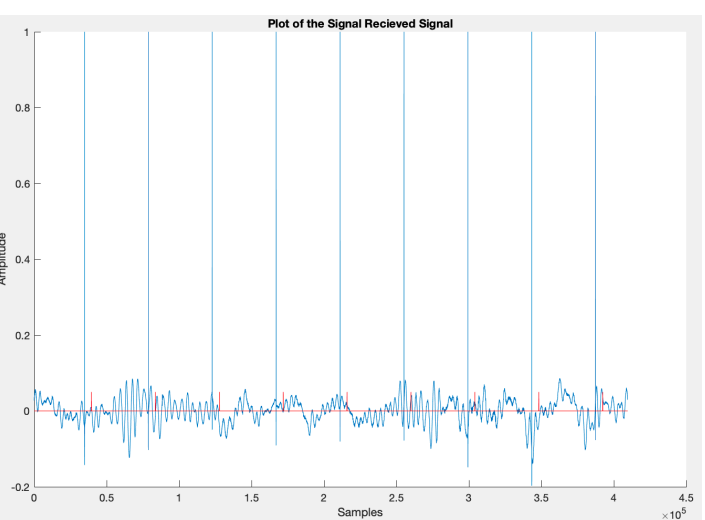

Figure 4.6

An overview map of the test site can be seen in figure 4.5. The manhole at point $A$ is where the pinger was mounted, and the sonar was put inside the pipe in point $\mathrm{B}$. The blue line represents the fresh water pipe we were testing in.

In figure 4.6 approximately 9 seconds of the recording from the sonar is plotted in blue. At first glance there is no apparent trace of the pinger pulse. To further investigate the data we start by calculating when the pinger signal is expected. At 3.5 degrees $\mathrm{C}$ and $7.3 \mathrm{bar}$, the speed of sound in fresh water is about $1420 \mathrm{~m} / \mathrm{s}$. Then the expected sample numbers of the pinger signals can be calculated. The results of these calculations are illustrated by the vertical red lines in the figure with an arbitrary amplitude of 0.05 . There is no obvious systematic spike in the recording when it is expected.

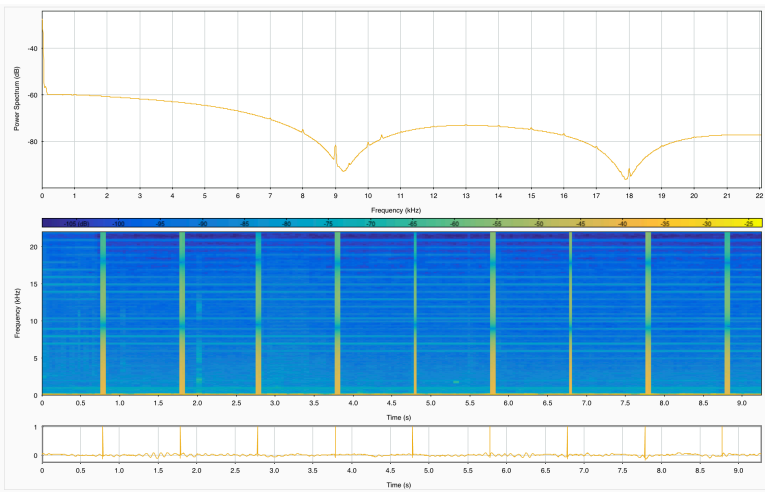

Figure 4.7
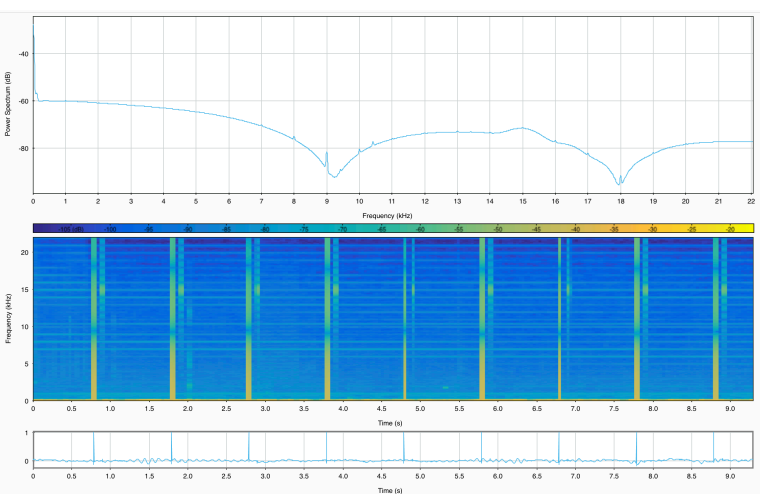

Figure 4.8

In figure 4.7 the recording can be seen in the Matlabs Signal analysis tool. There are no signs of the pinger pulse in the spectrogram or in the power spectrum. To visualize how an expected received pinger pulse would look like in this analysis a data overlay is created. The data overlay contains a $1 \mathrm{~ms}$ long $15 \mathrm{kHz}$ pulses with $10 \%$ of the theoretically expected amplitude. The recording with the data overlay in the Matlab Signal Analyzer tool can be seen in figure 4.8 . At just $10 \%$ of the expected amplitude with our parameters, the pulse is easily noticeable. This leads us to the conclusion that the pinger pulses is not received by the sonar for unknown reasons.

\section{Results}

From the start of this project a set of start requirements were set for the whole integrated system as well as the three subsystems. Every requirement was defined in such a way that a test would be able to confirm if it was met or not. The three subsystems were developed in design-build-test cycles until they individually were able to meet the start requirements. This 
was followed by tests of the subsystems working together or the system in its intended environment.

From the (4.1) leakage test we have learned that the sonar is able to detect leakages in near vicinity in the environment for which it is intended. We have also learned that it cannot detect leakages $156.3 \mathrm{~m}$ away, which matches with professionals experience and does not affect the working principle of concept in a negative way.

From the distance measurement test (4.2) we have learned that the integrated system is able to measure distances under water with a high accuracy. We have also learned that the waves sent out from the pinger have a high enough amplitude to theoretically reach a more than satisfactory distance.

From the distance measurement in the actual environment test (4.3) we have discovered that although all the subsystems meet all requirement, and the integrated system work, there is unknown difference between the prototyped pool environment and the actual pipe environment that renders our prototype ineffective. One or several of our assumptions of the pipe environment have we found to be wrong.

\section{Discussion}

The test of the integrated system in its intended environment did not work. Dynamic requirements were used to optimize for instance the battery in the sonar electronics and the choice of frequency of the pinger. Through testing we have learned that all the subsystems individually work and that the system as whole work in the environment of a pool. As a logical result of this the system should also work in other environments given that there is no critical difference between the two. To find plausible error sources for the distance measurement in the water distribution network, we will elaborate some differences with this environment compared to the environment of the successful measurements. We will also reflect upon and map out assumptions made which may be wrong.

Differences between the water distribution network environment and pool environment.

- The distance of the measurement is $148 \mathrm{~m}$ greater.

- Pressure of the environment is 7.3 bar greater.

- The temperature of the environment 7 degrees $C$ colder.

- The pulse has to traverse a polyethylene (PE) pipe with the inner dimension of $184.1 \mathrm{~mm}$

- The trajectory of the sound wave is indirect to the sonar. The signal needs to adjust direction either by reflection (or guiding) due to the bend in the pipe.

- The power source for the amplifier for the pinger was a car battery.

- The time from when the system was turned on to when the valuable data was recorded was greater.

- The pinger was mounted in an armature.

- The sonar was put in an armature.

- The sonar was standing up-right, instead of hanging in a bag.

Assumptions made

- The differences above does not affect the system to such an extent that the pinger pulse will not reach the sonar.

- There are no significant acoustic barriers between point A and B. 
- Sound pressure waves will decrease with a factor of $1 /$ distance, and all other decreasing factors such as absorption are neglectable at this pressure, frequency, temperature, salinity and range.

Our main hypothesis is that the car battery could not deliver enough current to the amplifier which could not be checked with our oscilloscope, combined with a greater loss of sound pressure by the inevitable reflections in the pipe walls. Both these factors weakened the signal to such an extent that the sonar could not register the signal. Testing our prototype integrated in the actual environment has given us an idea of which variables we should prioritize to test with prototyped test environments.

The method of participatory action research has been effective as the researcher gets firsthand knowledge of both the problem and solution space with an unparallel level of detail, when acting as the worker at the same time, as described in (Ottosson 2003). The potential downside is that the researcher may not use adequate amounts of resources to stop an reflect and alter implicit knowledge to pieces of explicit. In the current case the building author had had an academic environment around him, so that the reflection was frequent and the research method robust enough.

\section{Conclusion}

We have experienced that a prototype with every subsystem meeting its preset and emerged requirements, and that as whole proves to be working in the different relevant test environments, does not necessarily work in the actual environment. This illustrates not only insufficient knowledge of the problem space, but also of how the integrated solution interacts with the problem space. We believe that prototyping test environments to fit specific test variables and testing in them would have given valuable knowledge of the problem space (Winjum et al. 2017). But we also believe that only testing how the prototype integrated in the actual environment would have given knowledge of what test variables that should be prioritized. Testing in the intended environment may reveal wrong assumptions and limited knowledge that prototyped test setups never can. This because both the prototyped solution and the prototyped test environment are made with the same foundation of knowledge and assumptions. We, therefore, as an answer to the research question: "how to use prototyped test environments the best possible way in the early stage product development projects where the intended environment is complex?", suggest that the product developer should early-on actively alternate testing in the actual environment and prototyped test environments.

\section{References}

Blessing, Lucienne TM, and Amaresh Chakrabarti. 2009. DRM: A design reseach methodology: Springer.

Eisenhardt, Kathleen M. 1989. "Building theories from case study research." Academy of management review 14 (4):532-550.

Gerstenberg, Achim, Heikki Sjöman, Thov Reime, Pekka Abrahamsson, and Martin Steinert. 2015. "A Simultaneous, Multidisciplinary Development and Design JourneyReflections on Prototyping." International Conference on Entertainment Computing.

Kennedy, Brian M, Durward K Sobek, and Michael N Kennedy. 2014. "Reducing rework by applying set-based practices early in the systems engineering process." Systems Engineering 17 (3):278-296.

Kriesi, Carlo, Jørgen Blindheim, Øystein Bjelland, and Martin Steinert. 2016. "Creating dynamic requirements through iteratively prototyping critical functionalities." Procedia CIRP 50:790-795.

Norsk Vann, BA. 2017. Tilstandsvurdering av kommunale vann- og avløpstjenester. 
Ottosson, Stig. 2003. "Participation action research-: A key to improved knowledge of management." Technovation 23 (2):87-94.

Pahl, Gerhard, and Wolfgang Beitz. 2013. Engineering design: a systematic approach: Springer Science \& Business Media.

Pure. 2020. "SMARTBALL."

Schón, D. 1983. Ttu, Reflective Practitioner. Nueva York, Basic Books.

Schrage, Michael. 1996. Cultures of prototyping. ACM Press: New York.

Steinert, Martin, and Larry J Leifer. 2012. "'Finding One's Way': Re-Discovering a HunterGatherer Model based on Wayfaring." International Journal of Engineering Education 28 (2):251.

Sutcliffe, Alistair, and Pete Sawyer. 2013. "Requirements elicitation: Towards the unknown unknowns." 2013 21st IEEE International Requirements Engineering Conference (RE).

Winjum, Jardar, Andreas Wulvik, Jorgen AB Erichsen, Torgeir Welo, and Martin Steinert. 2017. "A heuristic approach for early-stage product development in extreme environments." 2017 International Conference on Engineering, Technology and Innovation (ICE/ITMC).

Yin, Robert K. 2017. Case study research and applications: Design and methods: Sage publications. 